\title{
Enhanced Performance with Brain Stimulation: Attentional Shift or Visual Cue?
}

\author{
James Cavanaugh, Bryan D. Alvarez, and Robert H. Wurtz \\ Laboratory of Sensorimotor Research, National Eye Institute, National Institutes of Health, Bethesda, Maryland 20982-4435
}

The premotor theory of visual spatial attention proposes that the same brain activity that prepares for saccades to one part of the visual field also facilitates visual processing at that same region of the visual field. Strong support comes from improvements in performance by electrical stimulation of presaccadic areas, including the frontal eye field and superior colliculus (SC). Interpretations of these stimulation experiments are hampered by the possibility that stimulation might be producing an internal visual flash or phosphene that attracts attention as a real flash would. We tested this phosphene hypothesis in the SC by comparing the effect of interchanging real visual stimuli and electrical stimulation. We first presented a veridical visual cue at the time SC stimulation improved performance; if a phosphene improved performance at this time, a real cue should do so in the same manner, but it did not. We then changed the time of SC visual-motor stimulation to when we ordinarily presented the veridical visual cue, and failed to improve performance. Last, we shifted the site of SC stimulation from the visual-motor neurons of the SC intermediate layers to the visual neurons of the superficial layers to determine whether stimulating visual neurons produced a larger improvement in performance, but it did not. Our experiments provide evidence that a phosphene is not responsible for the shift of attention that follows SC stimulation. This added evidence of a direct shift of attention is consistent with a key role of the SC in the premotor theory of attention.

Key words: colliculus; attention; visual; phosphene; macaque; stimulation

\section{Introduction}

Visual attention is the facilitation of visual processing of some stimuli over others. A shift of visual attention is usually accompanied by a saccadic eye movement to the attended area. Initial experiments in the superior colliculus (SC) showed that shifts of attention accompanying saccades made to a spot of light falling in the receptive fields of SC superficial layer neurons enhanced the visual responses of these neurons (Goldberg and Wurtz, 1972; Wurtz et al., 1980). Visual responses of neurons in several regions of cerebral cortex have subsequently shown enhancement with attention (for review, see Reynolds and Chelazzi, 2004). Improved visual processing is a hallmark of visual attention, and one hypothesis advanced to explain such improvements is the premotor theory of visual attention, which states that the same brain mechanisms that underlie the generation of saccades to one part of the visual field contribute also to the facilitation of visual processing in that part of the visual field (Rizzolatti, 1983; Moore et al., 2003). Thus, we might expect that the enhanced responses of visual neurons, whether in superficial SC or in cortex, may result from input from saccade-related neurons in the intermediate SC (Wurtz and Mohler, 1976) or from other saccade-related areas.

This premotor theory was first directly tested by Moore and

Received June 5, 2006; revised Sept. 8, 2006; accepted Sept. 10, 2006.

This work was supported by the intramural research program of the National Eye Institute. We thank Art Hays, John McClurkin, Altah Nichols, Tom Ruffner, and Mitchell Smith for their assistance.

Correspondence should be addressed to James Cavanaugh, Laboratory of Sensorimotor Research, National Eye Institute, Building 49, Room 2A50, 49 Convent Drive, Bethesda, MD 20982-4435. E-mail: jrc@|sr.nei.nih.gov. DOI:10.1523/JNEUROSCI.2376-06.2006

Copyright $\odot 2006$ Society for Neuroscience $\quad$ 0270-6474/06/2611347-12\$15.00/0
Fallah (2001) in experiments in which they stimulated the frontal eye field (FEF) with current too weak to evoke saccades but strong enough to reveal a modulation of the monkey's attention. Our own previous experiments on the SC (Cavanaugh and Wurtz, 2004), as well as those of Müller et al. (2005), showed that stimulating the saccade-related neurons in the intermediate layers of the SC produced an increase in performance consistent with the stimulation directly causing a shift in visual spatial attention. These stimulation experiments have moved the study of attention from simply correlating neuronal activity with behavior to demonstrating the causal relationship between them.

A major limitation of this advance, however, is that the electrical perturbation of neuronal activity that we take as producing a shift of attention may instead be producing an internal visual cue or "phosphene" (Brindley, 1982). In this "phosphene hypothesis," the stimulation generates a visual cue in the brain rather than directly shifting attention. Previous experiments (Moore and Fallah, 2001; Moore and Armstrong, 2003; Cavanaugh and Wurtz, 2004; Müller et al., 2005) have argued against the phosphene hypothesis, sometimes with the assistance of experimental controls (Müller et al., 2005). But this point requires more than just argument, because if the improved performance in these stimulation experiments is simply the result of attention following an internal visual cue, the experiments reveal nothing novel about the brain mechanisms underlying attention. Because this issue is so critical for understanding the neuronal mechanisms behind visual spatial attention, we specifically tested the phosphene hypothesis by comparing changes in performance produced by brain stimulation with those produced by veridical visual cues. 


\section{Materials and Methods}

Change detection task. The basic task underlying all of the experiments reported here involved change detection (Fig. 1). While the monkey fixated, a target appeared in the periphery. The target was a patch of $100 \%$ coherent random dot motion. The monkey's role was to determine, while continuing to fixate, whether the motion in the target changed direction. The target changed direction on $65 \%$ of trials, and the correct response to a change was a saccade to the target, whereas the correct response on trials when the target did not change was to remain fixating. Two distractors appeared with the target, also patches of dot motion, but the direction of motion in the distractors never changed. Additional details of the task procedures are provided below and in the previous report (Cavanaugh and Wurtz, 2004).

Collicular locations for experiments. We performed our tests of the phosphene hypothesis at eight different locations in the SC. We defined a collicular location as a series of penetrations targeting the same point in the visuotopic collicular map, yet spanning a series of depths, including superficial and intermediate layers. We performed the base experiment and the four tests described below at each SC location, but not in any fixed order. Each of these experiments lasted, on average, over $3 \mathrm{~d}$, with a range of 2-5 d, depending on the number of trials the monkey worked on a particular day. Results were pooled from the total duration of each experiment. The length of the experiments was determined by the need for a large number of catch trials requiring continued fixation to ensure that SC stimulation did not simply increase the frequency of saccades (false positives). In addition, because any of the three dot motion patches could be the target on any given trial, a single experiment typically lasted at least three days to obtain enough data to determine the significance of a positive effect while maintaining the unpredictability of the target. Adding the training trials given on days when the monkey's behavior was not adequate for the attention task, and time for searching for appropriate SC sites, investigation of each collicular location was spread over approximately a 6 week period. Below, we provide summaries of the base experiment and the four tests performed.

Base experiment. Our previous experiments (Cavanaugh and Wurtz, 2004) showed that stimulation of the SC intermediate layers improved performance on a change detection task in much the same way as did a veridical visual cue. Although we argued that SC stimulation was directly shifting attention in the same way that the monkey shifted attention in response to the veridical visual cue, the alternative hypothesis remained that attention was following a visual artifact of stimulation, a phosphene, rather than being shifted directly. Therefore, for direct comparison with the tests to follow, we repeated our initial stimulation experiment (Fig. $1 B$ ) at each collicular location to achieve a baseline effect of stimulation against which to compare the results obtained with each test. That is, we first established that stimulation improved change detection at that SC location. It was this improvement in performance at each location that we were able to quantitatively compare with the following tests of the phosphene hypothesis.

Testing the phosphene hypothesis. If one believes that the monkey's improved performance was a result of a phosphene acting as a cue, two major implications of accepting the phosphene hypothesis can be experimentally tested. The first implication is that the monkey immediately considered the phosphene as a visual cue, because SC stimulation had an immediate beneficial effect on performance after training with the veridical visual cue. Second, not only was the phosphene considered to be a cue, but the monkey could use this cue at the time of the change to improve detection in the same way the veridical visual cue was used before the patches of motion appeared. Our tests of the phosphene hypothesis (Fig. $1 C)$ primarily follow from these implications.

Test 1: veridical visual cue at time of change. First, if the monkey's attention follows a phosphene that occurs during the change, attention should follow a veridical visual cue in the same period and aid in detecting the change. To test this, instead of stimulating the SC at the time of the change, we presented the veridical visual cue with timing identical to that used for stimulation. If attention followed a phosphene, it should follow a veridical visual cue as well, and we should see the same type of improvement in performance.
Test 2: SC stimulation at premotion time. Second, because the monkey uses the veridical visual cue in the premotion period before the patches appear to direct attention to the location of the change, we would expect a putative phosphene in the same time period to direct attention as well. The monkey should learn to use a phosphene in this premotion period as cue to the location of the change, and we should see the same type of improvement in detection in both cases if stimulation, in fact, causes a phosphene.

Test 3: veridical visual cue at premotion time (single target). The original experiment using a veridical visual cue to direct attention (Cavanaugh and Wurtz, 2004) showed that the cue improved detection for any target location with which it was associated. In contrast, in our original stimulation experiment, we were only able to associate the cue with a single target location, as we were stimulating a single collicular location. What we did not know from the original study was how the monkey would behave when a veridical visual cue is associated with a single target location (like the stimulation) given the monkey's experience with having all target locations cued equally. For proper comparisons of behavior between using collicular stimulation premotion (test 2) and a veridical visual cue premotion, we needed to revisit our original experiment but this time, in the premotion period, cue only the target location associated with the current SC location. We would expect these results to match those of test 2 (stimulation premotion) if attention follows a phosphene induced by SC stimulation.

Test 4: stimulating the superficial layers of the SC. Our final test of the phosphene hypothesis was an attempt to make any putative phosphene more salient. If stimulating the intermediate layers produced a phosphene that attracted attention, then stimulating the more overtly visual neurons in the superficial layers of the SC should produce an even stronger phosphene, and a consequently stronger effect on detection.

Because there were multiple experiments to perform for each SC location, this required an average of more than $10 \mathrm{~d}$ of consistently low stimulation thresholds and overlapping visual fields (see inclusion criteria under SC Stimulation, below). Because the electrode was introduced afresh on each day, the stimulation threshold and field location criteria required for a full set of comparable stimulation experiments were not always met, so we were only able to obtain data for tests 2 and 4 at six SC locations, although no SC location is missing more than one test.

Although the general format of the base experiment and the four tests are outlined above, a detailed explanation of the experiments performed and the parameters used for the veridical visual cue and collicular stimulation follows, including specifications of timing.

SC stimulation. In each of two macaque monkeys (Macaca mulatta), a cylinder was implanted over the SC (centered on midline and angled at $42^{\circ}$ so that the electrode advanced rostrally), and neurons were recorded extracellularly using single tungsten microelectrodes and standard amplification, spike discrimination, and computerized data collection procedures as described in our previous report (Cavanaugh and Wurtz, 2004). All procedures were approved by the Institute Animal Care and Use Committee and complied with Public Health Service Policy on the humane care and use of laboratory animals.

After the SC had been located by single neuron recording, we positioned the electrode for stimulation of the intermediate layers. We concentrated on areas of the SC related to the visual field 5 to $20^{\circ}$ from the fixation point and inserted a guide tube that typically remained in place for the duration of the experiment at that SC location. We first advanced the electrode until reaching the visually responsive neurons in the SC superficial layers and then continued until reaching saccade-related neuronal activity. At that point, we passed a $60 \mu \mathrm{A}$, biphasic $200 \mathrm{~Hz}$ pulse train (biphasic pulse width, $0.1 \mathrm{~ms}$ ) through the electrode typically for $300 \mathrm{~ms}$ duration. We continued to slowly advance the electrode, and as soon as stimulation at $60 \mu \mathrm{A}$ began to evoke saccades, the current strength was lowered and the electrode advanced further until a current of $<10 \mu \mathrm{A}$ evoked saccades $50 \%$ of the time. The region of the field to which the saccade was directed was noted, and the center of one of the motion patches was placed at this point (see task specifics, below).

After determining the location of the stimulation site, we reduced the frequency of stimulation from $200 \mathrm{~Hz}$ to $70 \mathrm{~Hz}$ and increased the stimulation current to activate the target area in the SC without evoking 


\section{A Visual cue premotion}

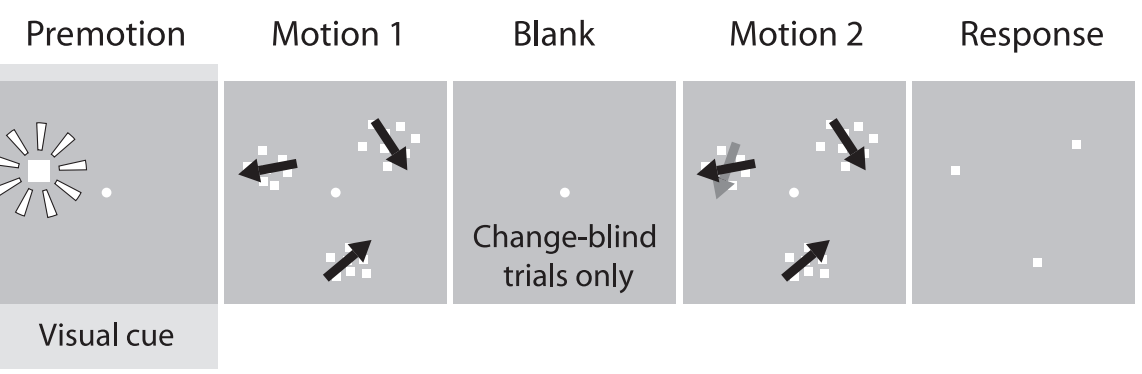

\section{B Stimulation during change}
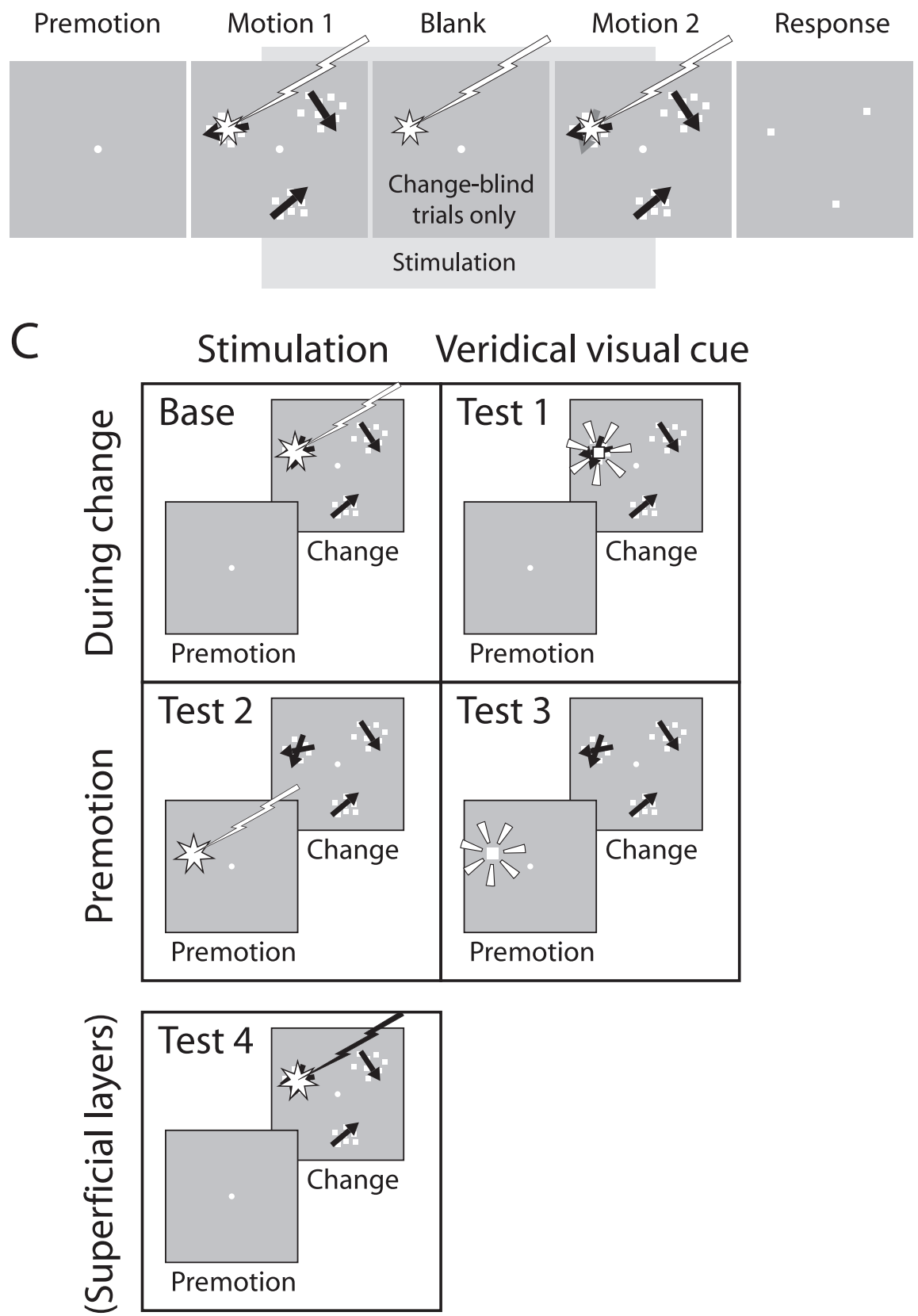

Figure 1. Change detection tasks. $A$, Shifting attention with a veridical visual cue. During initial fixation, a veridical visual cue on $50 \%$ of trials indicated where the target would appear. The cue disappeared, and three patches of random dot motion appeared: one target and two distractors. Motion in the patches commenced in two stages that were either contiguous (changevisible) or separated by a $150 \mathrm{~ms}$ blank (change-blind). $0 \mathrm{n} 65 \%$ of trials, the dots in the target patch changed direction between the first and second stages of motion. The monkey's task was to determine whether the target changed direction. If the motion in saccades. As reported previously by Glimcher and Sparks (1993), this lower frequency never evoked saccades even with a slight increase in current. In the intermediate layers, we increased the current level to approximately twice the threshold current, typically 15 or $20 \mu \mathrm{A}$. This was the current level used for the remainder of the day.

A new electrode was positioned through the implanted guide tube each day, and we required the stimulation to both meet the same threshold criteria and produce a saccade to the same region of the visual field as on the first day. If these two criteria were not met, the experiment at that location was terminated. Additionally, only those stimulation locations that showed an increase in performance from stimulating the intermediate layers of the SC (i.e., a positive base effect) were included in this study ( 8 of 10 locations), because our goal was to investigate possible alternative causes of a positive effect.

SC superficial layer stimulation. In addition to the tests in which we interchanged the veridical visual cue and the SC stimulation, we also compared the effect of stimulating the visual neurons in the SC superficial layers with that of stimulating the visual saccade neurons in the intermediate layers (test 4 ). To choose a stimulation site in the superficial layers, we again located the neurons with visual activity that indicated the electrode had entered the SC. We mapped the location of the visual field in which we could elicit visual responses using small stimuli with varying locations. We noted the center of this field and used this location for one of the motion patches. We again passed current through the electrode with the same stimulation parameters used for the intermediate layers, except this time we ensured that stimulation at $60 \mu \mathrm{A}$ did not evoke saccades. At some sites, we advanced the electrode to the intermediate layers until stimulation at $60 \mu \mathrm{A}$ did produce saccades and then retracted the electrode back up into the superficial layers until the sac-

\footnotetext{
the target changed direction, the correct response was a saccade to the target location. If the target did not change, the correct response was to remain fixating. The direction of motion in the distractor patches never changed. $\boldsymbol{B}$, Shifting attention with collicular stimulation. The sequence of stages in this task was nearly identical save for the absence of the veridical visual cue. Instead of the cue, the intermediate layers of the $\mathrm{SC}$ were stimulated just at the time when the monkey was required to attend to the target to determine whether it changed. Stimulation occurred on $50 \%$ of the trials when the target location overlapped the SC stimulation site. C, Tests of the phosphene hypothesis. The matrix illustrates interchanging of the veridical visual cue with collicular stimulation. Each diagram schematizes the base experiment or one of the four test experiments. Within a column (for the base experiment and tests 1-3), the timing of the stimulation (left column) or veridical visual cue (right column) is interchanged. Along a row, the timing remains the same, but the stimulation and veridical visual cue are interchanged at either the time of the change (top row) or in the premotion period (bottom row). The final test, stimulation of the $\mathrm{SC}$ superficial layers, appears separate from the matrix.
} 
cades disappeared. At some collicular locations, we attempted to study the effect of stimulating the superficial layers first so as not to damage them by passing the electrode through them repeatedly while advancing to the intermediate layers. As stated previously, we discarded data from superficial layer stimulation at two locations, because when we advanced the electrode into the intermediate layers, we did not find a significant effect of the SC stimulation, which was one of our criteria for inclusion.

In any case, the $>60 \mu \mathrm{A}$ threshold combined with the visual neuronal responses indicated that we were in the superficial layers, and results were similar no matter which procedure was used. In the superficial layers, we had no knowledge of the threshold current, so we used the same canonical stimulation current $(15-20 \mu \mathrm{A})$ there as well. This current level was then used for the rest of the experimental day. Stimulation was given at the same time (during the change) as it was in the base stimulation experiment.

Task specifics. We used the same task reported in our previous study along with slight variations of that task devised to test the phosphene hypothesis. Additional details of the task procedures are provided in the previous report (Cavanaugh and Wurtz, 2004). The same two monkeys were used in these experiments as in the previous experiments.

In the change-blind (CB) task used previously (Cavanaugh and Wurtz, 2004), we inserted a $150 \mathrm{~ms}$ blank just when the target might change direction, rendering the changes much more difficult to detect. The advantage of the change-blind task was that attention had a large effect on performance, because we showed how a veridical visual cue indicating the location of the target greatly improved change detection. The disadvantage was that it was difficult for the monkey to perform; therefore, easier trials with no blank between the two stages of motion had to be randomly interspersed to keep the monkey working. These changevisible $(\mathrm{CV})$ trials accounted for two-thirds of the data, so much of the recorded data from these experiments was not included in our original analysis. For the present investigation of the phosphene hypothesis, we needed to obtain as many trials as possible to compensate for the added permutations in the task necessary for testing the hypothesis. We therefore expanded the analysis to include both trials that placed a blank before the change of direction as in the previous report, which we refer to as change-blind trials, and those that did not, which we refer to as change-visible trials.

Figure $1 \mathrm{~A}$ shows the sequence of events in the original behavioral task when a veridical visual cue indicated the location of the target. Each trial began with the monkey fixating on a spot of light in the center of the screen. While the monkey fixated, a valid cue appeared on $50 \%$ of trials for $500 \mathrm{~ms}$, indicating which patch would be the target on that trial. On the remaining $50 \%$ of trials, no cue appeared, so the monkey had no previous knowledge of which patch was the target. The cue was a square spot of light $0.5^{\circ}$ across and centered on the future target location. After the cue disappeared, three patches of random dot motion, the target and two distractors, then appeared. The patches were equally distributed around the central fixation point. Motion in each patch was in a direction selected at random on each trial. The centers of the patches ( 7.5 to $15.0^{\circ}$ diameter) were placed between 8.5 and $20^{\circ}$ eccentric from the fixation point. After $750-1500 \mathrm{~ms}$, the first stage of motion ended, and the second stage of motion commenced. In $65 \%$ of trials, the direction of dot motion in the target changed, usually by $\sim 40^{\circ}$, from the first to second stage of motion. The second motion stage lasted at most 500-1000 ms. If the target had changed direction, the monkey received its reward (a drop of water) for making a saccade to the target (within a window of \pm 3.5 to $4.5^{\circ}$ of the target center) at any time during the second stage of motion. On the remaining $35 \%$ of trials, there was no change in the target, and the correct response on these trials with no change was to remain fixating for the duration of the second motion stage (recall that the distractor patches never change). Note that if the target did not change and there was no cue, the monkey would never know which patch was the target, but would get a reward anyway for continuing to fixate for the duration of the second stage of motion.

One-third of all trials were change-blind trials, incorporating the intervening 150 ms blank between the two stages of dot motion as in our original report. The remaining two-thirds of trials were change-visible trials with no intervening blank, so it was much easier for the monkey to detect changes in the target patch on these trials. These trials were more comparable with the direction change used in the attention experiments of Treue and Maunsell (1999). Note that the probability of the target changing (65\%) was independent of whether the trial was a change-blind or change-visible trial. So, to summarize, trials mainly consisted of two stages of dot motion, either separated by a blank (change-blind) or not separated by a blank (change-visible). The target patch changed direction only on some trials (65\%), and the distractor patches never changed. The monkey was rewarded with a drop of water by making a saccade to the target patch if it changed and continuing to fixate if it did not.

The base stimulation experiment (Fig. $1 B$ ) was identical to the behavioral task outlined above, except there was no veridical visual cue presented before the patches of motion appeared. We instead stimulated the intermediate layers of the SC just at the time that attention was required at the target patch to determine whether a change occurred (shaded stimulation epoch in Fig. 1B). Specifically, stimulation began $150 \mathrm{~ms}$ before the end of the first motion stage and lasted for $600 \mathrm{~ms}$. By keeping onset and duration of stimulation constant between change-visible and change-blind trials types, the duration of stimulation in the second stage of motion necessarily differed between trial types. This meant that stimulation lasted $300 \mathrm{~ms}$ into second stage of motion on change-visible trials and lasted only $150 \mathrm{~ms}$ into the second stage of motion on change-blind trials because of the duration of the blank. Note that because the SC stimulation site was location specific, one patch was placed overlapping the visual field representation of the collicular movement field, and stimulation was only possible when that patch was the target. In other words, stimulation occurred only on $50 \%$ of the trials when the target overlapped the movement field. Although some of our tests did not require SC stimulation, we still associated them with a collicular location by keeping one of the patches of dot motion centered over the visual field location corresponding to that particular SC location.

The four tests differed only slightly from the basic paradigms described above, and the logic of these differences is summarized in Figure 1C. Test 1 was identical to the base experiment, except that instead of SC stimulation, a veridical visual cue appeared centered on the target with the same timing and frequency of appearance as the stimulation in the base experiment. Test 2 was the same as the original behavioral task above except that instead of a veridical visual cue indicating the target in the premotion period, we stimulated the SC for $600 \mathrm{~ms}$ in the premotion period on $50 \%$ of the trials when the target overlapped the collicular movement field. Test 3 was identical to the original behavioral task with one exception: the veridical visual cue appeared in the premotion period on $50 \%$ of the trials only when the target overlapped the movement field associated with the SC location. Finally, test 4 was identical to the base experiment, except that we stimulated the superficial layers of the SC rather than the intermediate layers.

Note that for all tasks, the presence of stimulation (or of the veridical visual cue) was independent of trial type (change-blind or changevisible) and was also independent of whether the target changed or not. Also, recall that for all experiments in this study, SC stimulation (or the veridical visual cue) was associated only with the target overlapping the visual field location corresponding to the current SC location.

Performance measures. As in our original study, we assessed performance by comparing the frequency of hits and false positives on trials with the veridical visual cue (or with stimulation) to those trials without the manipulation. Hits were trials on which the monkey made a saccade to the target patch when it changed. The proportion of hits was the number of hits out of the total number of trials of the same type on which the target did change; the proportion ranged from 0 (never making a saccade to the target when it changed) to 1 (making a saccade to the target every time it changed). False positives for a given location were saccades to the patch at that location when there was no change in direction of motion in that patch (even if another patch was the target on that trial). The proportion of false positives also could range between 0 (never making a saccade to the patch when it did not change) and 1 (always making a saccade to the patch when it did not change). This meant that always making a saccade to a patch would yield $100 \%$ hits and $100 \%$ false positives for that patch and never making a saccade to a patch yielded $0 \%$ hits and $0 \%$ false positives for that patch. 
For each test at each SC location, hits and false positives were calculated separately for different conditions (e.g., trials with a cue and trials without a cue). We calculated hits and false positives only for the target patch overlapping the SC movement field, because this was the only patch location associated with SC stimulation (or with the cue). There were two primary reasons that we continued measuring hits and false positives separately instead of combining them into a single $d^{\prime}$ measure of change detection. First, because of our use of multiple patches to provide uncertainty as to the location of a possible direction change, there was a localization component to a correct response in addition to the detection component, so chance performance would yield $d^{\prime}$ values below zero. The unconventional values obtained for $d^{\prime}$ would therefore be difficult to interpret, if not somewhat misleading. Second, because of our use of collicular stimulation, it was critical to keep track of false positives explicitly, to rule out a direct motor effect of SC stimulation. Because $d^{\prime}$ combines hits and false positives into a single value, we would lose this necessary measure of a critical aspect of the monkey's performance.

To determine the significance of changes in hits and false positives, we used the standard conversion of differences between proportions into $\mathrm{z}$-scores. This is done by expressing the difference between two proportions $p_{1}$ and $p_{2}$ (this difference being, for example, the increase in the proportion of hits from stimulating the SC) in terms of the estimated SE of the proportion difference as follows:

$$
z=\frac{p_{1}-p_{2}}{\sigma_{p 1-p 2}},
$$

in which $\sigma_{p 1-p 2}$ is ideally the SE of the difference between the population means:

$$
\sigma_{p 1-p 2}=\sqrt{\sigma_{p 1}^{2}+\sigma_{p 2}^{2}}=\sqrt{\frac{p_{1}\left(1-p_{1}\right)}{n_{1}}+\frac{p_{2}\left(1-p_{2}\right)}{n_{2}}} .
$$

However, because our measurements only give us sample means for each proportion (for example, $p_{1}$ would be the proportion of hits with stimulation and $p_{2}$ the proportion of hits without stimulation), we use these proportions and the number of observations made to obtain them (for the example $p_{1}$ and $p_{2}$ above, $n_{1}$ would be the total number of stimulation trials on which the target changed, and $n_{2}$ would be the total number of nonstimulation trials on which the target changed) we can estimate $\sigma_{p 1-p 2}$ as follows:

$$
\sigma_{p 1-p 2} \approx \sqrt{\frac{p_{0}\left(1-p_{0}\right)}{n_{1}}+\frac{p_{0}\left(1-p_{0}\right)}{n_{2}}},
$$

in which the combined proportion estimate $p_{0}$ appropriately takes into account both proportions and their respective number of observations:

$$
p_{0}=\frac{p_{1} n_{1}}{n_{1}+n_{2}}+\frac{p_{2} n_{2}}{n_{1}+n_{2}} .
$$

We converted changes in the proportion of hits and false positives into $\mathrm{z}$-scores for both change-blind and change-visible trial types. We evaluated the significance of these changes in hits and false positives directly from the $\mathrm{z}$-scores, because there is a one-to-one correspondence between $\mathrm{z}$-score and significance. We determined the significance of overall effects across SC locations (for example, whether hits significantly increased overall with stimulation) using a nonparametric test (Wilcoxon sign-rank test for difference of the median).

Once we determined the magnitude of the change in performance in each test, we then needed to compare the results from each test with the results from the base stimulation task. At each collicular location, we again used z-scores to compare results from each test with the base SC stimulation task. This time, however, instead of expressing a difference in proportions (for example, hits with and without stimulation) in terms of the SE of the difference (see Eq. 1), we now needed to express the difference in the change in proportions (for example, comparing the change in hits from stimulation with the change in hits from presenting a veridical visual cue) in terms of the appropriate SE. For this, we use the following equation:

$$
z=\frac{\Delta p_{a}-\Delta p_{b}}{\sqrt{\sigma_{a}^{2}+\sigma_{b}^{2}}},
$$

in which $\Delta p_{a}$ is $p_{1}-p_{2}$ (as in Eq. 1) for test A (for example the change in hits $\Delta h$ from stimulation in the base experiment), and $\Delta p_{b}$ is $p_{1}-p_{2}$ for test B (for example, the change in hits $\Delta h$ obtained by presenting a veridical visual cue). The SE estimates $\sigma_{a}$ and $\sigma_{b}$ are the same SE estimates calculated in Equation 3 above. That is, $\sigma_{a}$ is just $\sigma_{p 1-p 2}$ for test A, and $\sigma_{b}$ is $\sigma_{p 1-p 2}$ for test B. Again, significances were obtained directly from the z-scores.

For examining trends in the difference in results between the base stimulation experiment and tests 1-4 across locations (for example, the significance of the overall difference in $\Delta h$ between the base experiment and test 1 ), we again ascertained the significance of the overall effects using a nonparametric test (Wilcoxon sign-rank test for difference from the median).

\section{Results}

\section{Primary effect of SC stimulation during the direction change (base experiment)}

We first determined the effect of stimulating the SC intermediate layers (Fig. $2 \mathrm{~A}$ ) to establish a baseline of the change in performance against which we could compare the tests of the phosphene hypothesis. We showed this attentional effect for both the change-blind trails, which we had used in our previous report, and for the change-visible trials, which we had not. Figure $2 B$ presents results from one example SC location where the target location overlapped the SC movement field, and the direction change to be detected was $40^{\circ}$. We separated the results of the randomly interleaved change-blind (Fig. $2 \mathrm{~B}, \mathrm{CB}$, open symbols) and change-visible trials (Fig. $2 B, \mathrm{CV}$, filled symbols). For each condition, we plotted the proportion of correct saccades to the target overlapping the SC movement field (hits) versus the proportion of incorrect saccades to that location (false positives). Gray symbols (Fig. 2 B, both open and filled) show performance on trials without $\mathrm{SC}$ stimulation. In the absence of any stimulation, the proportion of hits was higher for the change-visible (Fig. $2 \mathrm{~B}$, filled) than the change-blind (Fig. $2 \mathrm{~B}$, open) task, indicating the relative ease in detecting the change in motion without the intervening blank. Black symbols (Fig. $2 \mathrm{~B}$, both open and filled) show performance on trials when the SC was stimulated, and each connected pair of points shows the improvement in performance achieved by SC stimulation. The effect of stimulating the intermediate layers of the SC was similar to that seen by shifting attention with a veridical visual cue (Cavanaugh and Wurtz, 2004). That is, the improvement of performance at this target location was primarily an increase in hits, whereas the effect of stimulation on false positives was a slight reduction. Moreover, the improvement occurred for both the change-blind and change-visible conditions.

The magnitude of the improvement in performance in the base experiment at each of the eight SC locations is shown in Figure $2 C$. The effect of stimulation is shown as the change in hits from stimulation $(\Delta h)$ versus the change in false positives from stimulation $(\Delta \mathrm{fp})$. Data shown are for both for the change-blind trials (Fig. 2C, open symbols) and change-visible trials (Fig. 2C, filled symbols). The differences for the example location in Figure $2 B$ are outlined with squares. Across the sample, as for the example, the predominant effect of stimulating the intermediate layers of the SC was to increase the proportion of hits while leaving the proportion of false positives relatively unchanged. The mean change in hits was $15.2 \%(p<0.0001$; Wilcoxon sign-rank test $)$ 
A

\section{Base experiment: intermediate layer stimulation}

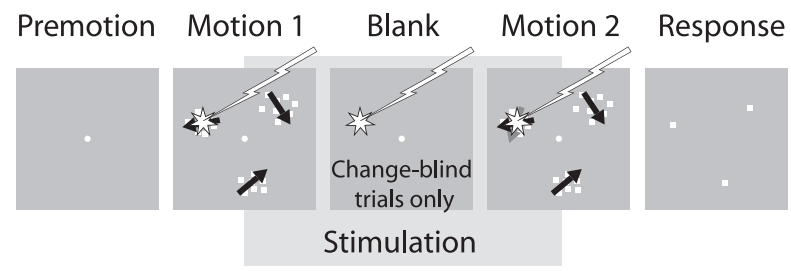

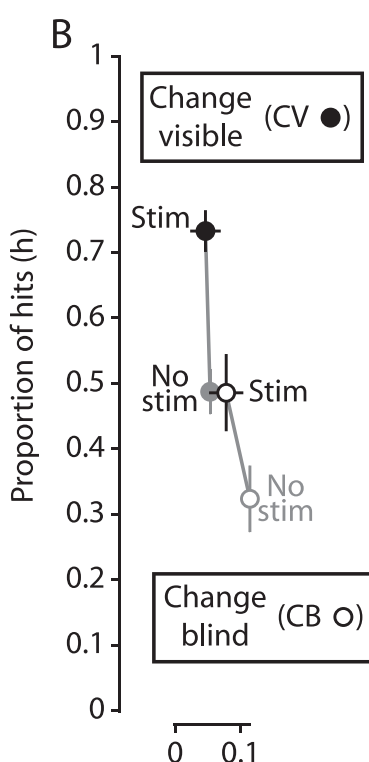

Proportion of false positives ( $\mathrm{fp}$ )
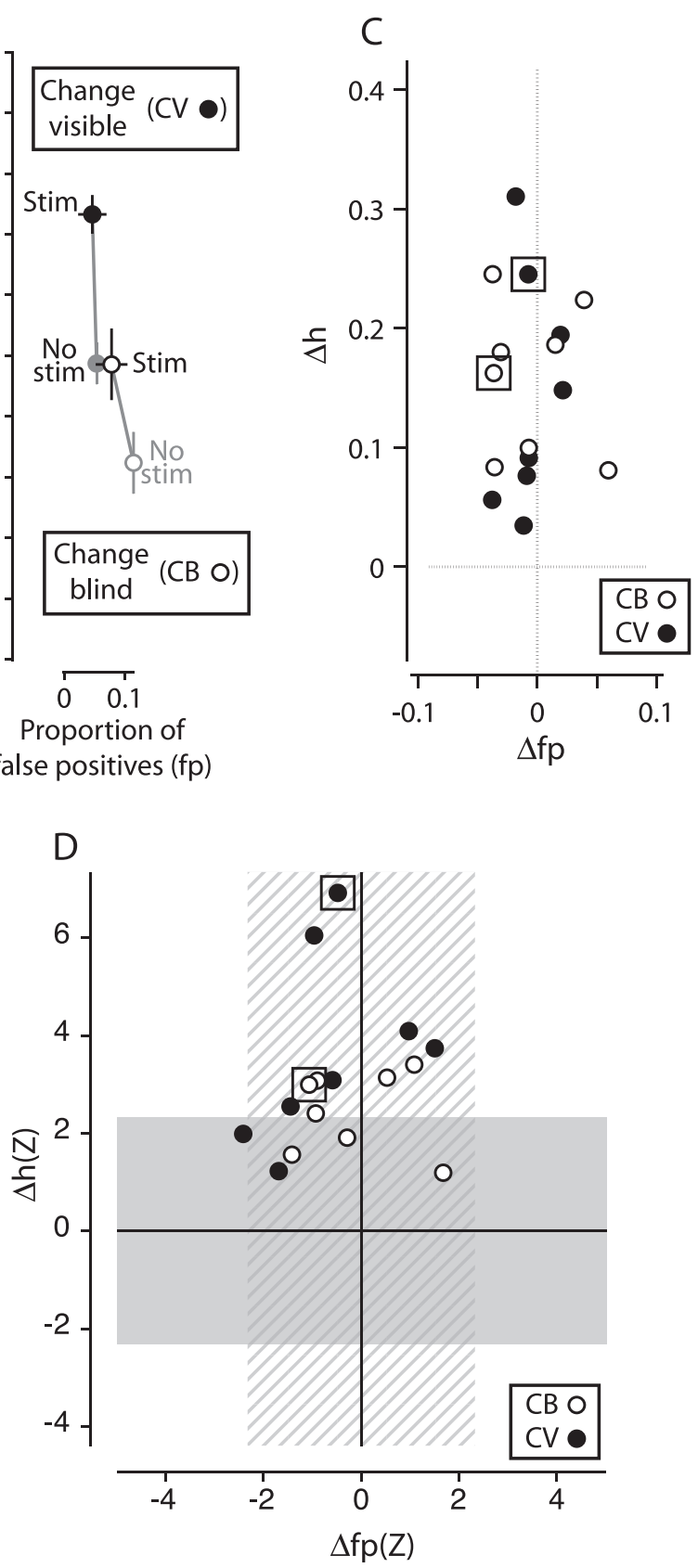

Figure 2. Base effects of $\mathrm{SC}$ stimulation on performance. $A$, In the base $\mathrm{SC}$ stimulation paradigm, we stimulated the superior colliculus around the time when the direction of dot motion in the target might change. Stimulation began $150 \mathrm{~ms}$ before the first stage of dot motion ended and lasted a total of $600 \mathrm{~ms}$. This was true for both the change-blind and change-visible tasks. $\boldsymbol{B}$, Sample result from a single collicular location. The proportions of hits are plotted against false positives. The gray symbols indicate the monkey's performance without collicular stimulation, and the black symbols show performance with stimulation. The connected pair of open symbols shows performance on change-blind trials, and the pair of filled symbols shows and the mean change in false positives was $-0.5 \%(p=0.240)$. Although in some subsequent experiments we observed differences in performance between the change-blind and changevisible tasks, these differences did not reach significance because of the spread of the data, so from here on we have pooled the two trial types when reporting population statistics (while continuing to visually differentiate the two tasks by symbol shading).

In Figure $2 D$, we converted the differences in proportions into $\mathrm{z}$-scores $[\Delta h(Z)$ and $\Delta \mathrm{fp}(Z)]$. Because z-scores are directly translatable into significances, we can indicate regions of significance by demarcating the region within $p>0.01$ (i.e., a z-score $<2.33$ ) on each axis. Points above or below the horizontal gray area represent significant changes in the proportion of hits, and points to the right or left of the vertical hatched area represent significant changes in false positives. We can see that SC stimulation facilitated the detection of the change in motion direction similarly in both the change-blind (Fig. 2D, open symbols) and the changevisible (Fig. $2 D$, filled symbols) cases so that both of these trial types can be used as a baseline for testing the phosphene hypothesis.

Using these data combined over the eight SC locations as a baseline for comparison, we then tested the phosphene hypothesis by performing a set of experiments designed to examine the interchangeability of collicular stimulation and a veridical visual cue. If collicular stimulation acts via an internal visual cue, the monkey should be able to use a phosphene and a veridical visual cue similarly, and we should see a corresponding similarity in the results. In contrast, differences in the results will cast doubts on the validity of the phosphene hypothesis.

\section{Test 1: replacing stimulation during the change with a veridical visual cue}

Our first test of the phosphene hypothesis was to replace the stimulation in our base experiments (Fig. 2) with a veridical visual cue (cueing only the motion patch associated with the SC location). The veridical visual cue appeared on the target patch with identical timing as the stimulation (i.e., during the potential change in motion) (Fig. $3 A$ ). The cue, like the stimulation, remained on during the $150 \mathrm{~ms}$ blank in change-blind trials. Because the veridical visual cue was $0.5^{\circ}$ across and the smallest target was $7.5^{\circ}$ in diameter, the cue appeared superimposed in the center of the target patch but the dot motion remained virtually unobstructed. If the attentional shift in our base results was a result of a phosphene being used as a visual cue, then the monkey must be able to use a visual cue at the time of the motion change (the stimulation period) to direct its attention. We would then expect a veridical visual cue during the change to enhance performance in the same manner (even if not with the same magnitude) as a putative phosphene, so the results from test 1 should be similar to the base results if we are to accept the phosphene hypothesis.

Figure 3, $B$ and $C$, shows how performance changed when we

the result from change-visible trials. C, Difference (stim - no stim) in hits and false positives ( $\Delta h$ and $\Delta \mathrm{fp}$, respectively) for each of eight collicular locations in two monkeys. Open symbols are from change-blind trials, and filled symbols are from change-visible trials. Positive values indicate that hits (or false positives) increased with stimulation. Results from the example SC location featured in $\boldsymbol{B}$ are indicated by squares. $\boldsymbol{D}, \mathbf{Z}$-scores for differences in performance shown in $C$. The shaded bands indicate regions where the data fail to achieve significance ( $p>$ 0.01). Points lying outside the horizontal gray area denote significant changes in hits, and points outside the vertical hatched area show significant changes in false positives. Again, the sample results from $\boldsymbol{B}$ are indicated by squares. 
A Test 1: single location cued at change

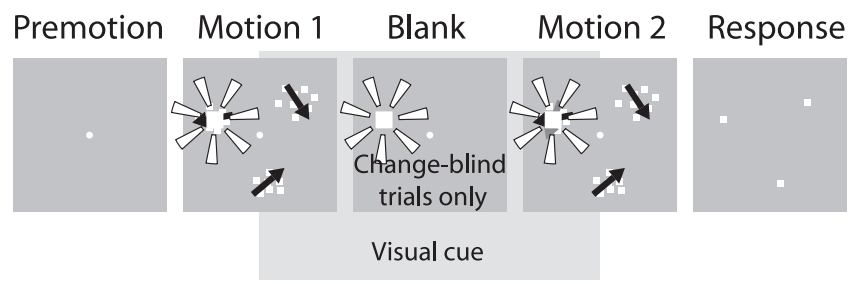

B

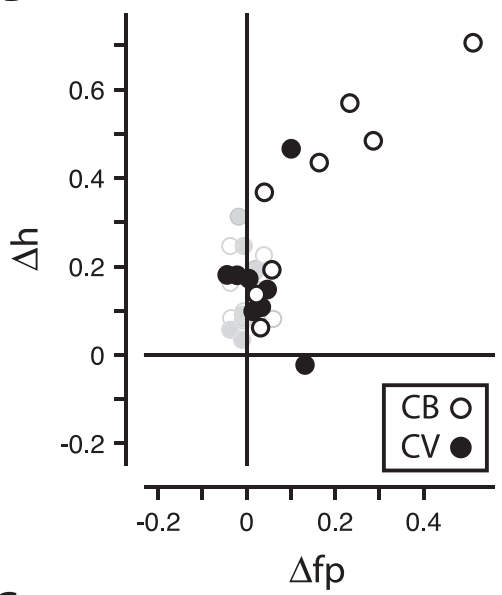

C

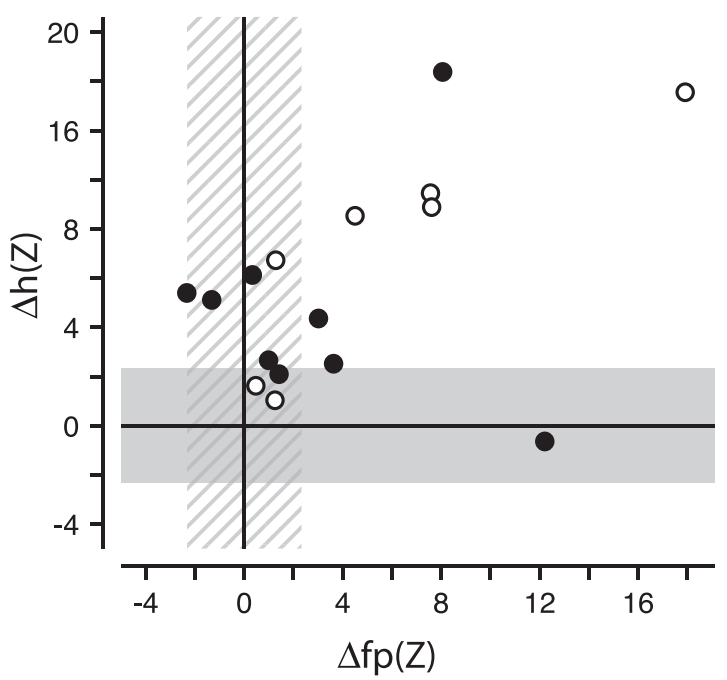

Figure 3. Test 1: veridical visual cue presented during the change. $\boldsymbol{A}$, The sequence of steps is similar to those in the base stimulation experiment (Fig. $2 A$ ), except that a veridical visual cue (for only one motion patch location) replaces the collicular stimulation on $50 \%$ of trials on which the target overlapped the visual field position for that SC location. The veridical visual cue came on at the same time as had the SC stimulation; we made no attempt to allow for the 40-50 ms time for a veridical visual cue signal to arrive at the $\mathrm{SC}$, because the cue still appeared early enough (150 ms before the change). $\boldsymbol{B}$, Change in the proportion of hits versus the change in the proportion of false positives when the veridical visual cue replaces $S C$ stimulation. Open symbols represent change-blind trials, and filled symbols represent change-visible trials. Base data from SC stimulation (from Fig. 2C) are in the background in gray. C, Differences in proportions plotted as the $z$-scores. Note that many individual changes lie outside the shaded regions and are therefore significant changes $(p<0.01)$.

presented a veridical visual cue on the target at the time of the change. As in the previous plot, filled symbols in Figure 3 represent the CV task, and open symbols represent the CB task. The changes in the proportions of hits $(\Delta h)$ and false positives ( $\Delta \mathrm{fp}$ ) are shown in Figure $3 B$, with the results from the base experiment shown in light gray for comparison. Hits showed a large increase of $26.8 \%$ ( $p=0.0005$; Wilcoxon sign-rank test), but false positives also increased $(+10.1 \%$; $p=0.0032)$. Figure $3 C$ shows the significance of individual changes. As in Figure 2, points outside the gray shaded band denote significant changes in hits, and points outside the hatched shaded band represent significant changes in false positives. Many of these changes were individually significant, as shown by the points lying outside one or both of the shaded bands. Compared with SC stimulation, the effect of the veridical visual cue on hits was $11.7 \%$ greater than the base SC stimulation data ( $p=0.030$; Wilcoxon sign-rank test), and the change in false positives was $10.6 \%$ greater than the base ( $p=$ $0.002)$. Note that although the changes in performance on change-blind and change-visible trials were not statistically different because of the small number of SC locations and the spread of the data, it is clear that it was on the change-blind trials that the greatest changes in hits and false positives occurred.

Overall, there were large significant increases in both hits and false positives, indicating that the veridical visual cue during the change caused the monkeys to make more saccades to the cued target, both correct and incorrect, rather than selectively increasing only correct saccades. Although hits increased more than false positives, implying perhaps some increase in performance (as might be expected from a valid visual cue), the increase in false positives is at odds with the base effect of SC stimulation. With respect to testing the phosphene hypothesis, cuing a single target during the change did not produce the same effect on performance as stimulating the SC during the change.

\section{Test 2: replacing the veridical visual cue with SC stimulation}

For our next test of the phosphene hypothesis, we again stimulated the intermediate layers but changed the timing of the stimulation to match that of the veridical visual cue in the premotion period. In our original behavioral experiments, the veridical visual cue appeared early in the trial (the premotion period), and the results showed that the monkey could definitely direct its attention using a visual cue before the stimuli appeared. We therefore reasoned that if the SC stimulation produced a phosphene, then this putative phosphene, if presented in the precue period, should at least produce some improvement in performance. Even if the supposed phosphene during the change acted by capturing attention with its onset rather than by the monkey directing attention to its location, the fact remains that the phosphene was visible and salient enough for the monkey to detect. If presented in the premotion period, the monkey should learn to use this visible, salient, and 100\% valid cue. Therefore, instead of stimulating at the time of the motion change as in the base experiment, we now stimulated at the exact time when we would have presented the veridical visual cue during psychophysics: while the monkey was fixating but before the patches appeared (Fig. 4A).

Figure 4, $B$ and $C$, shows the effect of stimulating the intermediate layers in the premotion period. The changes in performance from stimulation ( $\Delta h$ and $\Delta \mathrm{fp}$ ) are shown in Figure $4 B$, again with the base results in gray for comparison. Figure $4 C$ shows the results in the form of $\mathrm{z}$-scores. Any overall change in performance did not meet our criteria $(p=0.01)$ for significance. There was only a $3.7 \%$ increase in hits ( $p=0.034$; Wilcoxon sign-rank test) and a slight decrease in false positives $(-2.6 \% ; p=0.052)$. None of the changes in hits were significant in themselves (i.e., outside the gray region). When compared with the base data, the average change in hits from stimulation was $9.5 \%$ lower than base $(p=$ 0.0005 ) when stimulation occurred before the patches of motion 


\section{A Test 2: stimulation premotion}

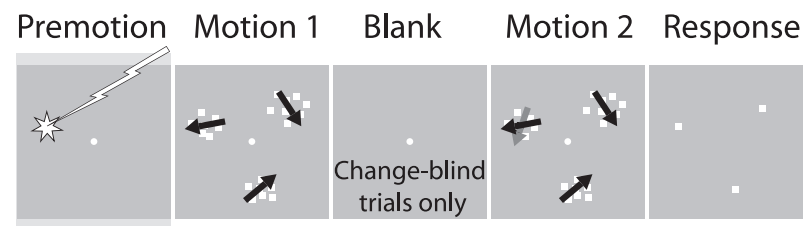

Stimulation
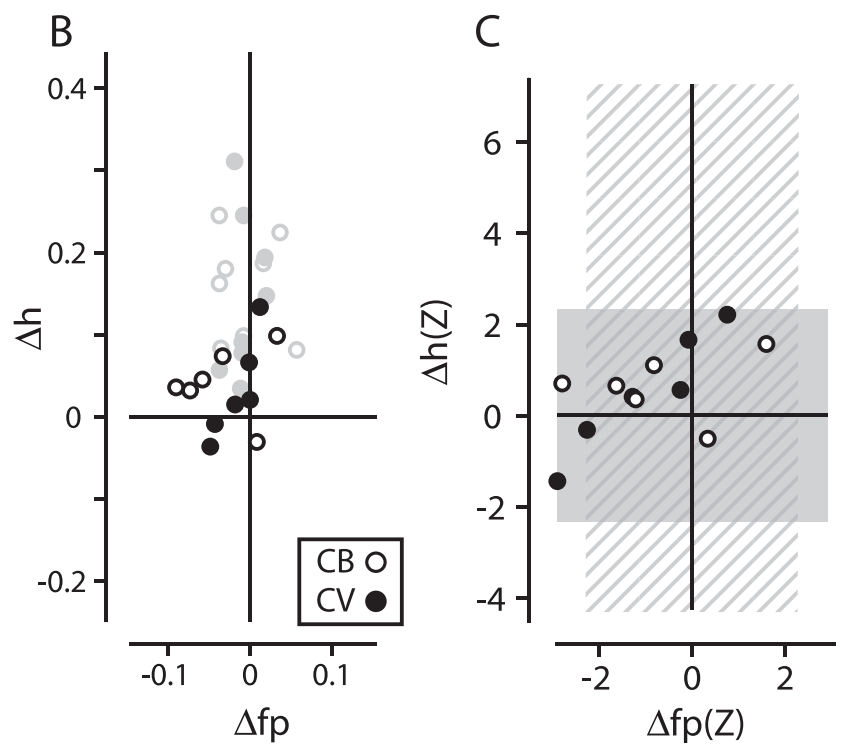

Figure 4. Test 2: SC stimulation in the premotion period. Same schema as in Figure 3. $\boldsymbol{A}$, Sequence of steps showing $S C$ stimulation of the intermediate layers in the premotion period (when the veridical visual cue normally appeared) rather than at the time of motion change. $\boldsymbol{B}$, Change in the proportion of hits versus the change in the proportion of false positives from stimulation. Open symbols represent change-blind trials, and filled symbols represent changevisible trials. The shaded symbols in the background are the data from the base experiment in Figure $2 C$. Note that receptive field location and stimulation threshold criteria were only achieved at six of the eight SC locations (see Materials and Methods). $\boldsymbol{C}$, The differences in proportions from $\boldsymbol{B}$ plotted as $z$-scores of the changes. The only significant ( $p \leq 0.01$ ) changes were two slightly significant reductions in false positives (points to the left of the hatched region).

appeared, but the slight decrease in false positives did not differ significantly from the base experiment (1.7\% lower than base; $p=0.15)$.

Whereas a veridical visual cue in the premotion period increased the proportion of hits (Cavanaugh and Wurtz, 2004), stimulating the intermediate layers of the colliculus with the same timing failed to produce a significant effect. Even if the phosphene differed in intensity or salience from the veridical visual cue, we would have expected some improvement in performance, because the phosphene generated by SC stimulation during the change (if we accept the phosphene hypothesis) did increase the proportion of hits. Even if the putative phosphene and the veridical visual cue are dissimilar enough that the monkey might not initially direct its attention to a phosphene, we would expect after thousands of trials in which the phosphene preindicates the target with $100 \%$ validity that the monkey would learn to direct its attention to the location indicated by the phosphene. But the monkeys were unable to use collicular stimulation before the patches appeared to improve performance, making it unlikely that stimulating the intermediate layers of the SC produced an internal visual cue. Again, the evidence does not support the phosphene hypothesis.

\section{Test 3: precuing a single target location}

To validly compare veridical visual cues and collicular stimulation, the next experiment addressed an asymmetry between the base SC stimulation paradigm and the veridical visual cue paradigm we used to obtain our original behavioral data. During stimulation experiments, we could only place one of the patches of dot motion at the visual field location corresponding to the SC stimulation site, because we were stimulating only one point in the SC. As a result, we could not associate SC stimulation with more than one of the target locations. In contrast, in our original psychophysical experiments (Cavanaugh and Wurtz, 2004), we directed attention with the veridical visual cue to any of the three targets. To make the premotion cue and SC stimulation results more comparable, we revisited our original behavioral paradigm with one important change: we associated the veridical visual cue in the premotion period with only the target location corresponding to the current collicular location. This was the only target location that was cued in test 3 .

Figure 5 shows how a veridical visual cue at a single location in the premotion period (Fig. $5 A$ ) changed the monkeys' performance. In Figure $5 B$, we plotted the effect of the veridical visual cue on the proportions of hits and false positives. The base stimulation data are again shown in gray for comparison. We observed an overall increase in false positives $(+13.5 \%$; $p=0.0013$; Wilcoxon sign-rank test) as well as hits $(+31.4 \%$; $p=0.0004)$, with many of these changes being individually significant (Fig. $5 C$, points falling outside the regions of nonsignificance). Compared with the base data, although hits overall were $16 \%$ greater in test 3 ( $p=0.0016)$, false positives were also greater than base $(+14 \%$ difference; $p=0.0016)$. Note that as in test 1 , it was on the change-blind trials that the greatest changes occurred, although the differences between the change-blind trials and the changevisible trials were not statistically significant.

Not only do these high false positive rates differ significantly from the base data, they also differ from the original psychophysical experiments (Cavanaugh and Wurtz, 2004). In these behavioral experiments, the veridical visual cue could be associated with any target location, and, as we had hoped, the monkey did not develop a response bias, as indicated by rare occurrence of false positives. When only one target was cued, the monkey did develop a response bias for that target, making more responses to the cued target, both correct (hit) and incorrect (false positive). This is the same type of behavior we observed when a single target location was cued during the change in test 1 (Fig. 3), and note that in both these tests that used a veridical visual cue, false positives were significantly greater than in the base experiment.

As a test of the phosphene hypothesis, cuing a single target in the premotion period (Fig. 5) produced drastically different results from stimulating the SC in the same period (Fig. 4). However, we might not reject the phosphene hypothesis if SC stimulation and a veridical visual cue in the same period produced effects that differed only in magnitude. But such a qualitative (as well as quantitative) difference in the results is in disagreement with the phosphene hypothesis and instead points toward the idea that stimulation of the intermediate layers of the SC directs attention through some means other than a phosphene.

\section{Test 4: stimulating the visual layers of the SC}

Our final manipulation was to stimulate in the superficial layers of the SC. Evidence exists of stimulation among the visual neu- 


\section{A Test 3: single location cued premotion}

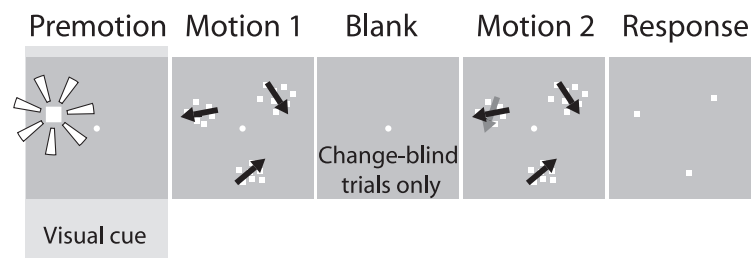

B

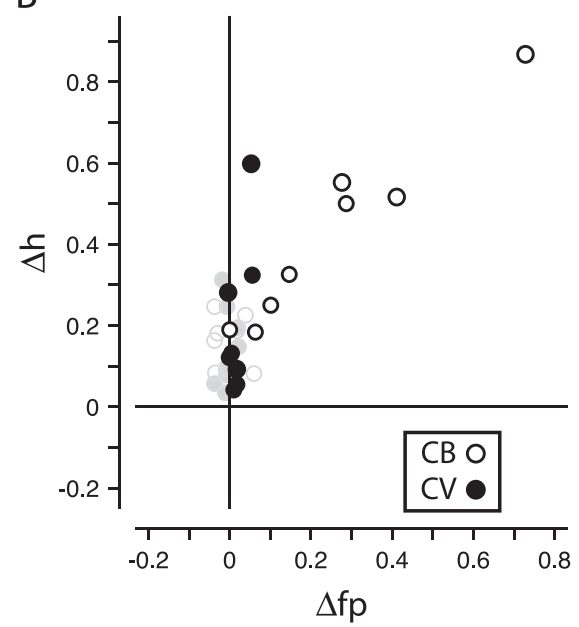

C

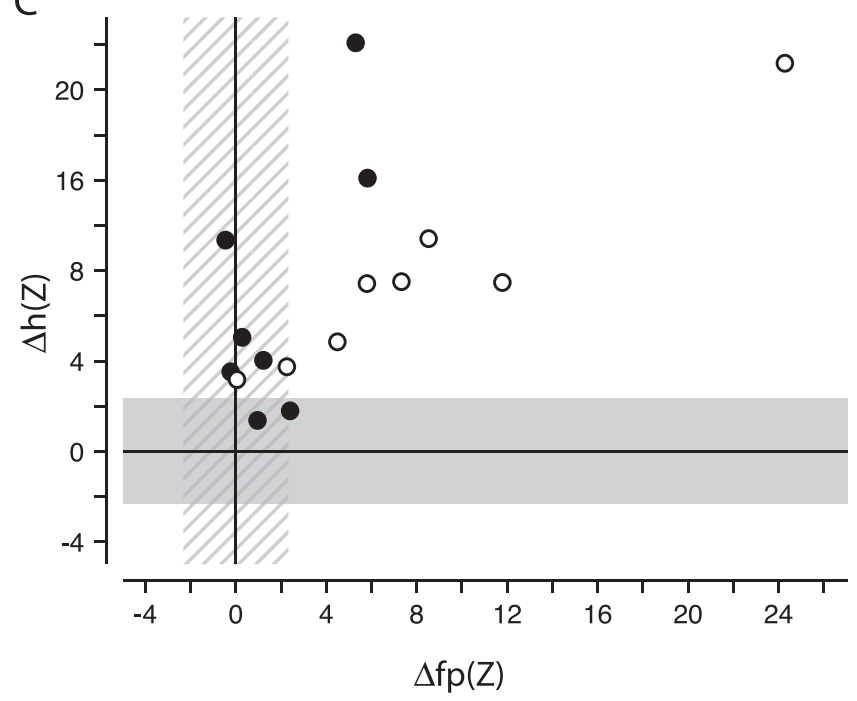

Figure 5. Test 3: cueing a single target location in the premotion period. Same schema as in Figures 3 and 4. A, The sequence of steps shows the veridical visual cue (for a single target location) in the premotion period. The cue appeared on $50 \%$ of trials when the target overlapped the visual field position for the current $\mathrm{SC}$ location. $\boldsymbol{B}$, Change in the proportion of hits versus the change in the proportion of false positives caused by the veridical visual cue. Open symbols represent change-blind trials, and filled symbols represent change-visible trials. Comparison data from the base experiment appear in gray in the background. C, Changes plotted as $z$-scores. Note that many changes in hits and false positives lie outside one or both shaded regions and are therefore significant changes.

rons in primary visual cortex producing phosphenes (Brindley and Lewin, 1968a,b; Dobelle and Mladejovsky, 1974; Dobelle et al., 1974; Schmidt et al., 1996; Lee et al., 2000), and we reasoned that stimulating the more overtly visual neurons in the superficial layers would make any visual consequence of the stimulation even more salient, produce a stronger cue, and thereby increase our chances of seeing a strong effect of stimulation. A stronger

\section{A Test 4: superficial layer stimulation}
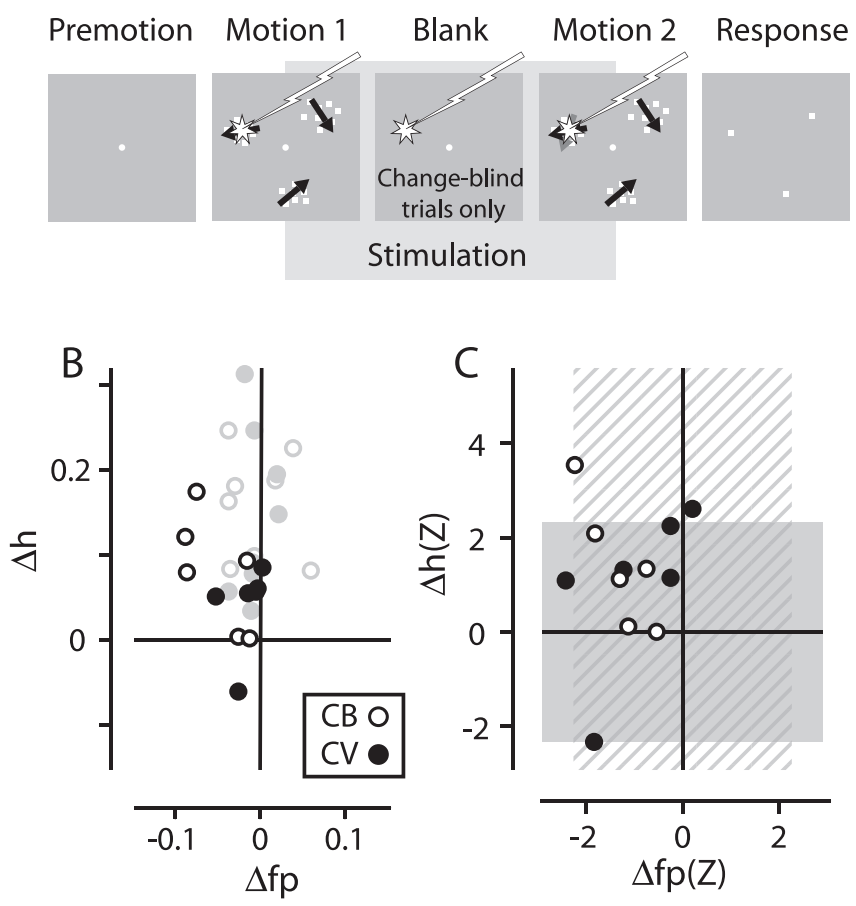

Figure 6. Test 4: stimulation of visual neurons in SC superficial layers. $\boldsymbol{A}$, Sequence of steps in the $\mathrm{SC}$ stimulation paradigm was identical to that used in the base experiment in the SC intermediate layers (Fig. 2A). B, Changes in hits and false positives (stim - no stim) for each of six SC locations in the superficial layers. Open symbols represent change-blind trials, and filled symbols represent change-visible trials. The base data from intermediate layer stimulation are shown in gray in the background for comparison. $\boldsymbol{C}$, z-scores for differences in performance shown in $\boldsymbol{B}$. Note the number of points falling in the shaded zones of no significant difference $(p>0.01)$.

cue should logically result in an even larger effect on performance if the phosphene hypothesis was correct.

Because comparison with our base experiment results was critical, we required the receptive fields at the stimulation sites in the superficial layers to be spatially consistent with the movement fields we obtained in the intermediate layers. We were able to meet this criterion at six of the eight SC locations we studied. When stimulating the superficial layers, we used the same stimulation parameters that produced attentional enhancement in the intermediate layers. Therefore, except for the depth of the electrode, the superficial layer stimulation experiments (Fig. 6A) were identical to the base experiments done in the intermediate layers (Fig. 2A).

For stimulation in the superficial layers of the SC, Figure $6 B$ shows the changes in performance at the target locations overlapping the visual receptive fields represented by the stimulation sites at each SC location. The base data from stimulating the intermediate layers are in the background in gray. Performance did not change much with superficial layer stimulation. There was a small significant effect on hits (6.0\% increase; $p=0.009)$, and false positives decreased slightly but significantly $(-3.4 \%$; $p=0.001)$. The $\mathrm{z}$-scores of these changes are shown in Figure $6 \mathrm{C}$. Note that in contrast to stimulating the intermediate layers, very few of the changes from superficial layer stimulation were significant (i.e., lying outside the shaded regions). The overall change in hits was significantly lower for superficial layer stimulation than for intermediate layer stimulation $(9.5 \%$ lower; $p<0.004)$. The changes in false positives were lower overall for superficial 

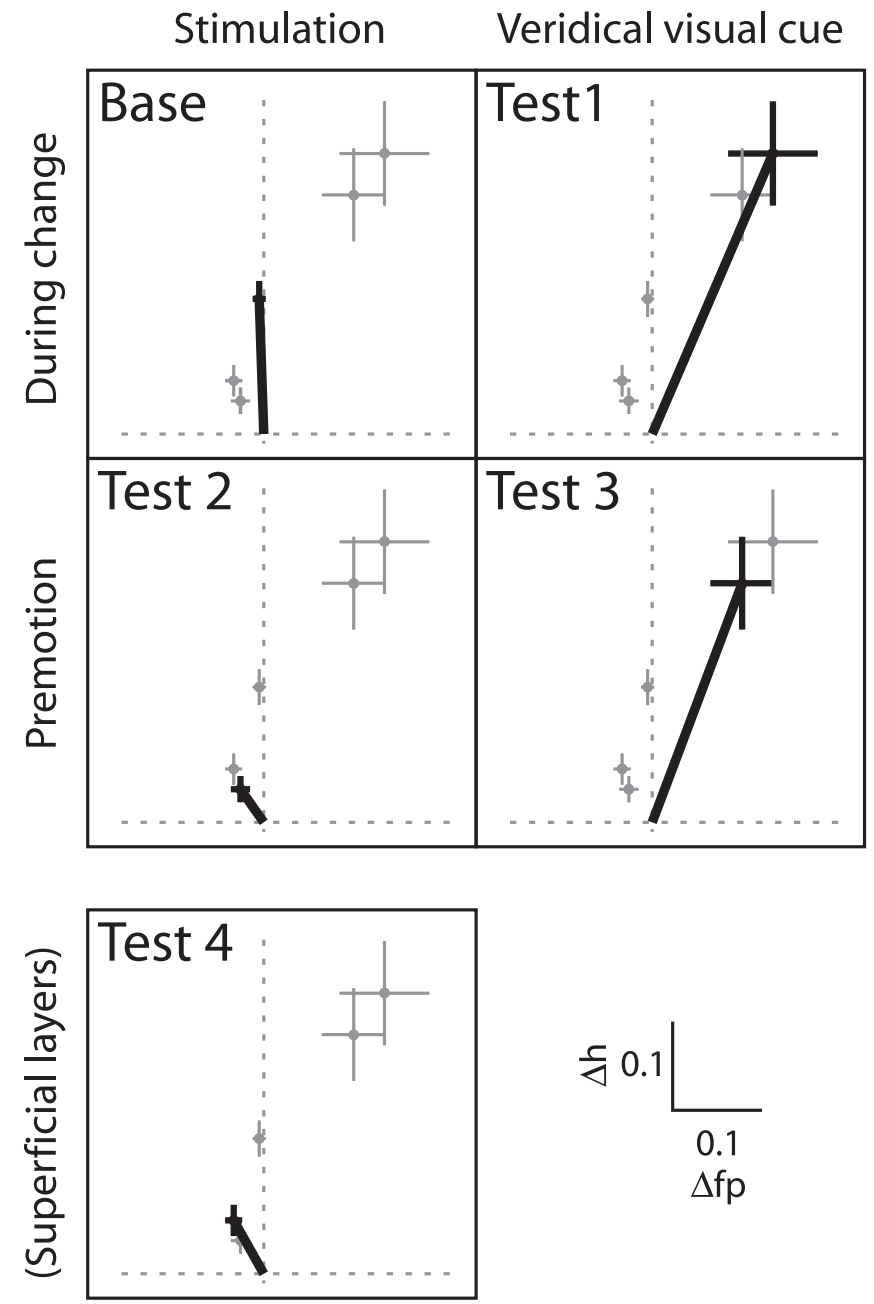

Figure 7. Summary of results in the base experiment and four tests of the phosphene hypothesis. Using the same scheme as in Figure $1 C$, within a column, the time of the stimulation (left) or veridical visual cue (right) were interchanged. Along a row, the timing remains the same, but the stimulation and veridical visual cues were interchanged at either the time of the change (top row) or at the premotion time (second row). Test 4 (superficial layer stimulation) appears separate from the matrix. For each of the base and four tests, we plotted the mean and SE for the change in hits $(\Delta h)$ versus the mean and SE of the change in false positives $(\Delta f p)$. Each point shows the mean for one experiment pooled over all SC locations. Gray dots and lines show means and SEs for each test and for comparison are repeated on all of the graphs. For a given experiment, the relevant mean and SE are plotted in black in the appropriate box and are further indicated by a black line from the origin to the mean for that experiment. The scale for the axes appears in the bottom right. None of our tests produced the same type of change in performance as did stimulation of the SC intermediate layers. See summary in Results for details.

layer stimulation than our base data (2.9\% lower) but not significantly so $(p=0.034)$.

We conclude that although stimulation of the visual neurons did improve performance slightly, the increase was not nearly as large as that obtained in the intermediate layers and very different from that obtained with a veridical visual cue in the same period (test 1) (Fig. 3), counter to the expectations of the phosphene hypothesis.

\section{Summary of results}

The results of our tests of the phosphene hypothesis are summarized in Figure 7, organized in the same format used in Figure $1 C$ to outline the different experiments. For the base and each test, we plotted the mean and SE of the changes in hits (ordinate of each graph) versus the mean and SE of the changes in false positives (abscissa) pooled over all of the SC locations tested. That is, each point represents the mean effect for the base experiment or one of the tests over all SC locations. For ease of comparison, the mean and SE for all of the experiments are shown in all of the graphs in gray. The relevant mean and SE on each graph is plotted in black and further highlighted by the black line connecting it to the origin. In contrast to the base results, the veridical visual cues (tests 1 and 3) always produced an increase in false positives as well as hits. This implies a potential increase in performance from the cue but one that is qualitatively different from the base result. The remaining tests using stimulation (tests 2 and 4) also differed from the base result but showed a decrease rather than an increase in false positives, again indicating a very different effect between presenting a veridical visual cue and stimulating the SC, regardless of timing.

Note that in Figure 7 (for the base experiment and tests 1-3), within a column, it is the timing of the stimulation or veridical visual cue that is interchanged, whereas along a row, the timing remains the same, but the stimulation and veridical visual cue are interchanged at the indicated time. If SC stimulation produced some kind of internal visual cue, we would expect the patterns of change from stimulation to be at least qualitatively similar to those obtained from using a real visual cue. That is, the results across a row should look similar, even if with different magnitudes because of the relative strength of a putative phosphene, but they do not. Moreover, a veridical visual cue gave remarkably similar results no matter when it appeared (Fig. 7, right column). However, the results from the base experiment and test 2 (Fig. 7, left column) tell a different story. Collicular stimulation had significantly different effects depending on when it was given, in contrast to what happened with a veridical visual cue. We believe that the comparisons among the full pattern of results in Figure 7 give convincing evidence that the improvement in performance from SC stimulation is not the product of attention after an internal visual cue.

\section{Discussion}

The premotor theory of visual spatial attention posits that the same brain activity directing saccades to one part of the visual field also improves visual processing in that part of the visual field. We tested the premotor theory in the intermediate layers of the SC, because here saccadic movements are exquisitely mapped onto the visual field, a substantial population of neurons discharge before saccades, and this neuronal activity builds up long before the saccade occurs, during fixation, when visual processing should be enhanced by shifts of attention. These characteristics form an ideal substrate for shifting attention in accordance with the premotor theory. In our present and previous experiments (Cavanaugh and Wurtz, 2004), stimulation of these saccade-related neurons enhanced visual processing by improving detection of a change in direction of motion. This is consistent with the idea that neuronal activity leading to a shift of the fovea also produced a shift of attention to the same part of the field. However, these same results are also consistent with what we have called the phosphene hypothesis, namely, that attention followed a stimulation-evoked visual flash to that part of the visual field.

\section{Phosphenes from stimulation}

The concern about phosphenes introduced by electrical stimulation in attention experiments arises primarily from the verbal reports of phosphenes by humans during stimulation of primary 
visual cortex, first reported by Brindley and Lewin (1968a,b) and then corroborated by subsequent experiments (Dobelle and Mladejovsky, 1974; Dobelle et al., 1974; Schmidt et al., 1996; Lee et al., 2000). In contrast, no such carefully executed experiments have been done for SC, and therefore, evidence for phosphenes there is sparse (Nashold and Slaughter, 1969; Tasker et al., 1980). In the monkey, evidence has accumulated that they can detect V1 stimulation at low currents as if they were seeing a phosphene (Bartlett et al., 2005; DeYoe et al., 2005; for review, see Tehovnik et al., 2005), bringing into question the effects of stimulating SC. The major similarity between stimulation of monkey V1 and SC is that stimulating either V1 (Tehovnik et al., 2003) or SC (Robinson, 1972; Stryker and Schiller, 1975) induces saccades to the part of the visual field represented by the neurons at the site of stimulation. However, stimulation of V1 leads to saccades with latencies no shorter than $50 \mathrm{~ms}$ (Tehovnik et al., 2003), consistent with stimulation affecting a sensory stage of processing, as expected when activating V1. In contrast, saccade latencies from stimulating SC intermediate layers under comparable behavioral conditions are $\sim 25 \mathrm{~ms}$ (Stryker and Schiller, 1975), consistent with stimulation affecting an output stage of visual motor processing. Thus, stimulation in V1 or SC produces saccades, but by activation of different ends of the visual-motor sequence, so it would not be surprising if activation of one end produces phosphenes and the other does not. An even more striking difference is that suprathreshold (i.e., evoking saccades) stimulation of V1 competes with visual targets as if two visual stimuli were being presented (Tehovnik et al., 2004). In contrast, suprathreshold stimulation of SC intermediate layers evokes mandatory saccades independent of any added visual targets. Thus, although limited comparisons can be made between the experiments in V1 and SC, they do lend support to the idea that phosphenes result from V1 stimulation but not from SC stimulation.

\section{Interchanging SC stimulation and veridical visual cues}

Although we argue against phosphenes being responsible for the shifts in attention we observed in our experiments, we believed these and similar studies would be reinforced with more direct evidence against attention following a visual cue during SC stimulation. Therefore, we addressed the phosphene hypothesis by testing whether the veridical visual cue and SC stimulation acted in the same way when interchanged. As summarized in Figure 7 and detailed in Results, we substituted the veridical visual cue and SC stimulation at their corresponding times. Neither substitution produced the same selective improvement in performance, counter to the hypothesis that the SC stimulation produces a phosphene used as a visual cue.

An alternate interpretation for the failure of SC stimulation to act like a visual cue, however, is that SC stimulation did produce a phosphene, but it was simply too dissimilar from the veridical visual cue for the monkey to generalize from one to the other. But this interpretation does not fit with the two monkeys' behavior during initial stimulation experiments. In these experiments, when we first stimulated the SC intermediate layers, both monkeys showed improved performance in the first sessions. If the stimulation was acting as a degraded visual stimulus, why was the monkey in that case able to immediately use SC stimulation instead of a veridical visual cue to improve its performance? Put another way, why were the monkeys able to use SC stimulation as a visual cue within the first few hundred trials but were unable to shift between using a real visual stimulus and a phosphene in our current experiments even after many thousands of trials? This argues strongly against a phosphene being responsible for the shifts of attention that we conclude derive from SC stimulation.

\section{Simulating SC visual neurons}

SC superficial layer neurons have strong visual responses rather than the clear saccade-related activity and limited visual responses of intermediate layer neurons. If stimulating SC produces a phosphene, stimulation of visual neurons there should produce stronger phosphenes, and the putative visual cue from stimulating SC superficial layers should therefore be more effective. We tested this by stimulating adjacent regions of SC intermediate and superficial layers related to the same part of the visual field. As summarized in Figure 7 (test 4), there was a slight improvement in detection from superficial layer stimulation but less than from stimulating the intermediate layers. These results again are counter to those expected from the phosphene hypothesis. A parsimonious explanation for the small effect on performance from superficial layer stimulation is that the current is spreading to intermediate layers, or activating neurons connecting to the intermediate layers [as demonstrated in the rat (Lee et al., 1997; Isa et al., 1998)], with the intermediate layers being responsible for the shift of attention.

That superficial layer stimulation does produce some improvement in performance and, however, might give some clues as to the route by which SC activity influences visual processing. The improved performance we observe is on a motion task, so we assume the ultimate effect of SC stimulation is cortical, because visual motion processing in the primate is concentrated in cortex. The prominent route from the intermediate layers of SC to cortex is through the medial dorsal thalamus to the FEF, so consequently any attentional effect might be acting through the FEF. If some effect can also be obtained from the superficial layers, this might implicate the prominent projection from SC through the inferior pulvinar directly to visual cortex (Benevento and Fallon, 1975; Benevento and Rezak, 1976), including the motion area MT (Adams et al., 2000; Shipp, 2001; Weller et al., 2002). It is possible that the effects of intermediate layer stimulation result from an as yet unverified projection from the intermediate layers to the superficial layers, hypothesized to explain the attentional augmentation of visual responses in the superficial layers (Wurtz and Mohler, 1976). In any case, the effect on performance from stimulating the superficial layers provides information, however speculative, about the routes by which SC influences visual processing in cortex.

\section{Rejecting the phosphene hypothesis}

An exact perceptual comparison of a visual cue and a phosphene is not possible, but the evidence favors SC stimulation producing a direct shift of attention rather than an internal visual cue. We therefore reject the phosphene hypothesis and regard our original interpretation of the stimulation effects as most reasonable; SC stimulation directly produces a shift of attention.

Our rejection of the phosphene hypothesis certainly applies to the SC stimulation experiments of Müller et al. (2005), suggesting that their results as well are not because of a phosphene, as they concluded from their own analysis. Our arguments might apply to the experiments of Moore and Fallah (2001) who stimulated $\mathrm{FEF}$, an area where the neurons also exhibit visual and saccaderelated activity. Because FEF neurons with saccadic activity are embedded in a much larger population of other neurons with visual rather than visual motor activity, it is difficult to determine whether acceptance or rejection of the phosphene hypothesis in monkey FEF should depend on our results in SC or the aforemen- 
tioned phosphene studies in V1. However, one would expect stimulation of human FEF with transcranial magnetic stimulation to reveal phosphenes if they were present, but to our knowledge none have been reported (Ruff et al., 2006).

Regardless, these experiments cannot tell us whether the shift in attention is primarily a product of FEF or of SC, or a combination of the two. However, despite the possible pathways by which these signals enhance visual processing, the preponderance of the evidence points to the conclusion that attention does not follow a phosphene but rather shifts directly in response to SC stimulation.

\section{References}

Adams MM, Hof PR, Gattass R, Webster MJ, Ungerleider LG (2000) Visual cortical projections and chemoarchitecture of macaque monkey pulvinar. J Comp Neurol 419:377-393.

Bartlett JR, DeYoe EA, Doty RW, Lee BB, Lewine JD, Negrao N, Overman Jr WH (2005) Psychophysics of electrical stimulation of striate cortex in macaques. J Neurophysiol 94:3430-3442.

Benevento LA, Fallon JH (1975) The ascending projections of the superior colliculus in the rhesus monkey (Macaca mulatta). J Comp Neurol 160:339-361.

Benevento LA, Rezak M (1976) The cortical projections of the inferior pulvinar and adjacent lateral pulvinar in the rhesus monkey (Macaca mulatta): an autoradiographic study. Brain Res 108:1-24.

Brindley GS (1982) Effects of electrical stimulation of the visual cortex. Hum Neurobiol 1:281-283.

Brindley GS, Lewin WS (1968a) The visual sensations produced by electrical stimulation of the medial occipital cortex. J Physiol (Lond) 194:54-55P.

Brindley GS, Lewin WS (1968b) The sensations produced by electrical stimulation of the visual cortex. J Physiol (Lond) 196:479-493.

Cavanaugh J, Wurtz RH (2004) Subcortical modulation of attention counters change blindness. J Neurosci 24:11236-11243.

DeYoe EA, Lewine JD, Doty RW (2005) Laminar variation in threshold for detection of electrical excitation of striate cortex by macaques. J Neurophysiol 94:3443-3450.

Dobelle WH, Mladejovsky MG (1974) Phosphenes produced by electrical stimulation of human occipital cortex, and their application to the development of a prosthesis for the blind. J Physiol (Lond) 243:553-576.

Dobelle WH, Mladejovsky MG, Girvin JP (1974) Artifical vision for the blind: electrical stimulation of visual cortex offers hope for a functional prosthesis. Science 183:440-444.

Glimcher PW, Sparks DL (1993) Effects of low-frequency stimulation of the superior colliculus on spontaneous and visually guided saccades. J Neurophysiol 69:953-964.

Goldberg ME, Wurtz RH (1972) Activity of superior colliculus in behaving monkey. II. Effect of attention on neuronal responses. J Neurophysiol 35:560-574.

Isa T, Endo T, Saito Y (1998) The visuo-motor pathway in the local circuit of the rat superior colliculus. J Neurosci 18:8496-8504.

Lee HW, Hong SB, Seo DW, Tae WS, Hong SC (2000) Mapping of functional organization in human visual cortex: electrical cortical stimulation. Neurology 54:849-854.

Lee PH, Helms MC, Augustine GJ, Hall WC (1997) Role of intrinsic synap- tic circuitry in collicular sensorimotor integration. Proc Natl Acad Sci USA 94:13299-13304.

Moore T, Armstrong KM (2003) Selective gating of visual signals by microstimulation of frontal cortex. Nature 421:370-373.

Moore T, Fallah M (2001) Control of eye movements and spatial attention. Proc Natl Acad Sci USA 98:1273-1276.

Moore T, Armstrong KM, Fallah M (2003) Visuomotor origins of covert spatial attention. Neuron 40:671-683.

Müller JR, Philiastides MG, Newsome WT (2005) Microstimulation of the superior colliculus focuses attention without moving the eyes. Proc Natl Acad Sci USA 102:524-529.

Nashold BS, Slaughter DG (1969) Effects of stimulating or destroying the deep cerebellar regions in man. J Neurosurg 31:172.

Reynolds JH, Chelazzi L (2004) Attentional modulation of visual processing. Annu Rev Neurosci 27:611-647.

Rizzolatti G (1983) Mechanisms of selective attention in mammals. In: Advances in vertebrate neuroethology (Ewert J-P, Capranica R, Ingle DJ, eds). New York: Plenum.

Robinson DA (1972) Eye movements evoked by collicular stimulation in the alert monkey. Vision Res 12:1795-1808.

Ruff CC, Blankenburg F, Bjoertomt O, Bestmann S, Freeman E, Haynes JD, Rees G, Josephs O, Deichmann R, Driver J (2006) Concurrent TMSfMRI and psychophysics reveal frontal influences on human retinotopic visual cortex. Curr Biol 16:1479-1488.

Schmidt EM, Bak MJ, Hambrecht FT, Kufta CV, O’Rourke DK, Vallabhanath P (1996) Feasibility of a visual prosthesis for the blind based on intracortical microstimulation of the visual cortex. Brain 119:507-522.

Shipp S (2001) Corticopulvinar connections of areas V5, V4, and V3 in the macaque monkey: a dual model of retinal and cortical topographies. J Comp Neurol 439:469-490.

Stryker MP, Schiller PH (1975) Eye and head movements evoked by electrical stimulation of monkey superior colliculus. Exp Brain Res 23:103-112.

Tasker RR, Organ LW, Hawrylyshyn P (1980) Visual phenomena evoked by electrical stimulation of the human brain stem. Appl Neurophysiol 43:89-95.

Tehovnik EJ, Slocum WM, Schiller PH (2003) Saccadic eye movements evoked by microstimulation of striate cortex. Eur J Neurosci 17:870-878.

Tehovnik EJ, Slocum WM, Schiller PH (2004) Microstimulation of V1 delays the execution of visually guided saccades. Eur J Neurosci 20:264-272.

Tehovnik EJ, Slocum WM, Carvey CE, Schiller PH (2005) Phosphene induction and the generation of saccadic eye movements by striate cortex. J Neurophysiol 93:1-19.

Treue S, Maunsell JH (1999) Effects of attention on the processing of motion in macaque middle temporal and medial superior temporal visual cortical areas. J Neurosci 19:7591-7602.

Weller RE, Steele GE, Kaas JH (2002) Pulvinar and other subcortical connections of dorsolateral visual cortex in monkeys. J Comp Neurol 450:215-240.

Wurtz RH, Mohler CW (1976) Organization of monkey superior colliculus: enhanced visual response of superficial layer cells. J Neurophysiol 39:745-765.

Wurtz RH, Goldberg ME, Robinson DL (1980) Behavioral modulation of visual responses in the monkey: stimulus selection for attention and movement. In: Progress in psychobiology and physiological psychology (Sprague JM, Epstein AN, eds), pp 43-83. New York: Academic. 